\title{
COVID-19 Vaccine: Concerns and Comfort, Who Should Take It?
}

\author{
Mohammad Khalid 1,2,3* \\ ${ }^{1}$ Consultant Pulmonologist/Intensivist, King Faisal Specialist Hospital \& Research Center Riyadh, KSA \\ ${ }^{2}$ Professor of Medicine, AlFaisal University College of Medicine, KSA \\ ${ }^{3}$ Faculty, John Hopkins University Hospital, USA
}

COVID-19 impact on humanity is going to be long lasting from health to loss of human life, it's economic constrains will continue to have devastating impact for a long time to come.

The successful outcome and launch of COVID-19 vaccine, has brought a sigh of relief to suffering humanity, with the shared happiness also has emerged the conspiracies of foul play and insecurity of potential harm.

The question is, who should get the vaccine and is it safe? There are several aspects that need some consideration: The vaccine is a new technology mRNA coated vaccine from the spike protein of the virus [1]. The way the vaccine will work in simple understanding the coated mRNA vaccine will access cell cytoplasm with the help of coating substance, once inside the cells cytoplasm it will generate the virus spike protein from host cell cytoplasm, vaccine is a live mRNA virus protein code unlike common flu vaccine which is non-living protein, once mRNA generates viral spikes protein on the cell surface it will be read as foreign by the body immune system and it will generate antibody defense system against it. In this process the cell will also go through lysis by the antibody generated. It is said the mRNA will not cross the nucleus and does not incorporate itself into the DNA the building block of cell genetic material|[2]. It certainly will cause plasma protein replication of mRNA to generate same sequenced proteins, how this replication effects cell protein is not very clear.

How many cells will get involved in this process? Will vaccine mRNA cross blood brain barrier? Would neurons have similar process? Would they die in process of antibody generation? Could nervous system cellular death cause longterm dementia or worsen existing dementia? Since neurons cannot be regenerated, could cell death of myocardium cause myocardial fibrosis in the long term? Above all, how would it effect the reproductive system, and would it lead to birth defects since cytoplasm would be altered by vaccine mRNA of the virus? There is no known definite process of mNRA metabolism, or a definite disintegration process and it is not known if proteins from its debris get incorporated in DNA. How long this antigen-antibody process will continue? Could it lead to autoimmune disorders or worsen the already existing autoimmune disorders? Could it cause lymphoprolifer- ative disorders? Some case reports of lymphadenopathy in some vaccine recipients have already been reported [3].

All of these questions will need to be answered as the post vaccination data emerges.

So, with above concerns, who should get the vaccine? To answer this, help could be obtained from national data of morbidity and mortality in COVID-19 disease it seems during this pandemic less than 1000 people died in age group 24 years and less. The impact is minimal, even less than many already known illnesses. The age group 24-60 has second lowest mortality impact, the worst affected group is 60 and above with the highest mortality, more than 150,000 died in this group so far [2]. We believe that this is where the impact of vaccine could make a difference.

In my opinion the vaccine will best serve its purpose if we vaccinate frontline workers, healthcare professionals and people above age 60. People in age group 25-60 and are suffering from immune suppression, obesity and other chronic illnesses labeled as high-risk group should receive vaccination and volunteer vaccination to healthy $24-60$ year age group. Age group 24 or below no vaccination.

Which vaccine should people take? A common question asked. Currently available vaccines are Pfizer and Moderna COVID-19 mRNA vaccines. The above mentioned concerns are related to these vaccines they are based on new mRNA technology, other vaccines in pipeline are Sino-vac (Chinese vaccine), Oxford vaccine and Astrazeneca vaccines. These

*Corresponding author: Professor Dr. Mohammad Khalid, Consultant Pulmonologist/Intensivist, King Faisal Specialist Hospital \& Research Center Riyadh, KSA; Professor of Medicine, AlFaisal University College of Medicine; Faculty, John Hopkins University Hospital, USA

Received: September 30, 2020

Accepted: March 03, 2021

Published online: March 05, 2021

Citation: Khalid M (2020) COVID-19 Vaccine: Concerns and Comfort, Who Should Take It?. Vaccine Res Dev 2(1):6-7 
are more traditional old fashioned technology based vaccine made on same principles as influenza vaccines. Above concerns most likely do not apply to these vaccines. People can choose if they have choice either to go with new mRNA vaccine technology or vaccines which may become available from Oxford, Astrazeneca or Sino-vac, the old traditional way generated vaccines [4].

In our opinion, this will provide the best outcome in controlling the spread, mortality and morbidity benefits, will also prevent massive cost loss as well as alleviate unfounded concerns. This may protect young population from any perceived future birth defects/cardiac complications possible dementia and other associated perceived complications. Patient with autoimmune disorders and existing dementia preferentially should be given non-mRNA based vaccine to prevent deterioration in their status.

\section{References}

1. National Center for Immunization and Respiratory Diseases (2020) Understanding and Explaining mRNA COVID-19 Vaccines.

2. Alberts B, Johnson A, Lewis J, et al. (2002) From DNA to RNA. In: Molecular Biology of the Cell. ( $4^{\text {th }}$ edn), Garland Science, USA.

3. Polack FP, Thomas SJ, Kitchin N, et al. (2020) Safety and Efficacy of the BNT162b2 mRNA Covid-19 Vaccine. N Engl J Med 383: 2603-2615.

4. National Center for Health Statistics (2020) Weekly Updates by select Demographic and Geographic Characteristic, Deaths involving Coronavirus disease2019 (COVID-19), pneumonia, and influenza reported to NCHS by sex and age group. United States.

DOI: $10.36959 / 669 / 746$ 